\title{
Using ARToolKit for 3D Hand Position Tracking in Mobile Outdoor Environments
}

\author{
Wayne Piekarski and Bruce H. Thomas \\ Wearable Computer Laboratory \\ School of Computer and Information Science \\ University of South Australia \\ Mawson Lakes, SA, 5095, Australia \\ \{wayne,thomas\}@cs.unisa.edu.au
}

\begin{abstract}
This paper describes how we have used the ARToolKit to perform three degree of freedom tracking of the hands, in world coordinates, which is used to interact with a mobile outdoor augmented reality computer. Since ARToolKit generates matrices in camera coordinates, if errors occur during the calibration process, it is difficult to extract out real world coordinates. We discuss the problem of making ARToolKit generate world coordinates, and the solutions we developed to meet the requirements for our tracking system.
\end{abstract}

\section{Introduction}

We have been performing research into mobile outdoor augmented reality, with examples such as the Tinmith-Metro application [2] shown in Figure 1. In these applications, we have been developing new user interaction techniques, as traditional desktop devices such as mice and keyboards do not work well in mobile outdoor environments. In order to interact with the complex modelling features of Tinmith-Metro, we have developed a glove which is the primary input device for the system. Unfortunately, traditional tracking technology, such as magnetic, ultrasonic, and acoustic systems, are designed for indoor use and are not suitable for placement onto a mobile outdoor backpack. To solve these problems, we have developed a cheap and low cost 3 DOF (degrees of freedom) hand tracker based on the ARToolKit libraries, allowing us to use a video camera mounted on the head to track the motion of the hands of the user as real world X, Y, Z values.

The ARToolKit libraries [1] were developed to support the tracking of simple fiducial markers, allowing applications to appear to place 3D objects onto these markers, and then viewed on a display device - an example is the ARToolKit simpleTest application. It should be noted that while the ARToolKit generates 6DOF matrices for each fiducial marker that are sent to OpenGL, these matrices are relative to a special distorted camera frustum model, and not usually in real world coordinates. If the calibration model for the camera is not perfect (the ARToolKit calibration process does not always generate good results) then extra errors are introduced into the results and it is unusable for tracking.

For more information, as well as videos of the system in use outdoors, readers are invited to visit our web site at http://www.tinmith.net
This paper discusses problems with calibration and getting accurate results for use as a tracker, and then discusses the solutions that we used to make the tracker work to our requirements.

\section{Gloves and video cameras}

We use a set of custom designed gloves to control the system, using metallic contacts on the fingertips, thumb, and palm to detect finger presses. We created two ARToolKit fiducial markers, each $2 \mathrm{~cm} \times 2 \mathrm{~cm}$ in diameter, and glued them to the thumbs of the glove (see Figure 1). We placed the markers on the thumb because this allows us to perform finger presses without moving the position of the marker. The size of the markers is the most appropriate size given that the position tracking is always within arms reach of the head mounted camera.

We use a PGR Firefly camera mounted on the user's head to capture the video and then pass it to ARToolKit. The camera has good dynamic range and can capture the fiducial markers in direct sunlight, at sunset, and in complete darkness with an extra light on the camera. Since the tracker operates in real world coordinates, it is possible to mount the camera anywhere appropriate on the body and transform the coordinates using a scene graph. The camera used for tracking does not have to be the same as that used for the AR overlay, and allows users watching from external VR views to see the locations of the cursors floating in front of the user's avatar.

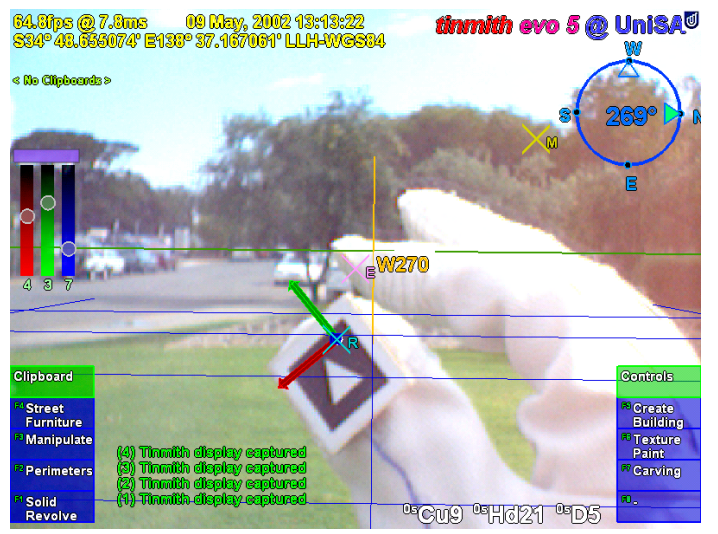

Figure 1 - Tinmith immersive augmented reality display, with gloves and $2 \mathrm{~cm}^{2}$ fiducial markers, and 3D overlaid $\operatorname{red}(\mathrm{X}) /$ green $(\mathrm{Y})$ / blue $(\mathrm{Z})$ cursor axes 
